\title{
Behavior Analysis of Human Locomotion in the Real World and Virtual Reality for the Manufacturing Industry
}

\author{
PHILIPP AGETHEN, Daimler AG and Ulm University \\ VISWA SUBRAMANIAN SEKAR, Daimler AG \\ FELIX GAISBAUER, Daimler AG and Ulm University \\ THIES PFEIFFER, CITEC, Bielefeld University \\ MICHAEL OTTO, Daimler AG and Ulm University \\ ENRICO RUKZIO, Ulm University
}

This is the author's version. While the content is identical, formatting differs from the published version. Follow the DOI to receive the published version.

\begin{abstract}
With the rise of immersive visualization techniques, many domains within the manufacturing industry are increasingly validating production processes in virtual reality (VR). The validity of the results gathered in such simulations, however, is widely unknown - in particular with regard to human locomotion behavior. To bridge this gap, this paper presents an experiment, analyzing the behavioral disparity between human locomotion being performed without any equipment and in immersive virtual reality while wearing a head-mounted display (HMD). The presented study $(n=30)$ is split up in three sections and covers linear walking, non-linear walking and obstacle avoidance. Special care has been given to design the experiment so that findings are generally valid and can be applied to a wide range of domains beyond the manufacturing industry. The findings provide novel insights into the effect of immersive virtual reality on specific gait parameters. In total, a comprehensive sample of $18.09 \mathrm{~km}$ is analyzed. The results reveal that the HMD had a medium effect (up to 13\%) on walking velocity, on non-linear walking towards an oriented target and on clearance distance. The overall-differences are modeled using multiple regression models, thus allowing the general usage within various domains. Summarizing, it can be concluded that VR can be used to analyze and plan human locomotion, however, specific details may have to be adjusted in order to transfer findings to the real world.
\end{abstract}

CCS Concepts: • Human-centered computing $\rightarrow$ Human computer interaction (HCI); User studies; User models;

Additional Key Words and Phrases: Virtual reality, human locomotion behavior, gait analysis, comparison, user studies

ACM Reference format:

Philipp Agethen, Viswa Subramanian Sekar, Felix Gaisbauer, Thies Pfeiffer, Michael Otto, and Enrico Rukzio. 2018. Behavior Analysis of Human Locomotion in the Real World and Virtual Reality for the Manufacturing Industry. ACM Transactions on Applied Perception 15, 3, Article 20 (July 2018), 19 pages.

https://doi.org/10.1145/3230648

\section{INTRODUCTION}

The manufacturing industry is currently facing an increasing demand for mass-customization which is gradually leading to growing numbers of different product variants. As a result, production planning methods have to be profoundly revised in order to meet the changing conditions. In particular, the product and its corresponding

Permission to make digital or hard copies of all or part of this work for personal or classroom use is granted without fee provided that copies are not made or distributed for profit or commercial advantage and that copies bear this notice and the full citation on the first page. Copyrights for components of this work owned by others than ACM must be honored. Abstracting with credit is permitted. To copy otherwise, or republish, to post on servers or to redistribute to lists, requires prior specific permission and/or a fee. Request permissions from permissions@acm.org.

(C) 2018 Association for Computing Machinery. 1544-3558/2018/07-ART20 \$15.00

https://doi.org/10.1145/3230648

ACM Transactions on Applied Perception, Vol. 15, No. 3, Article 20. Publication date: July 2018. 

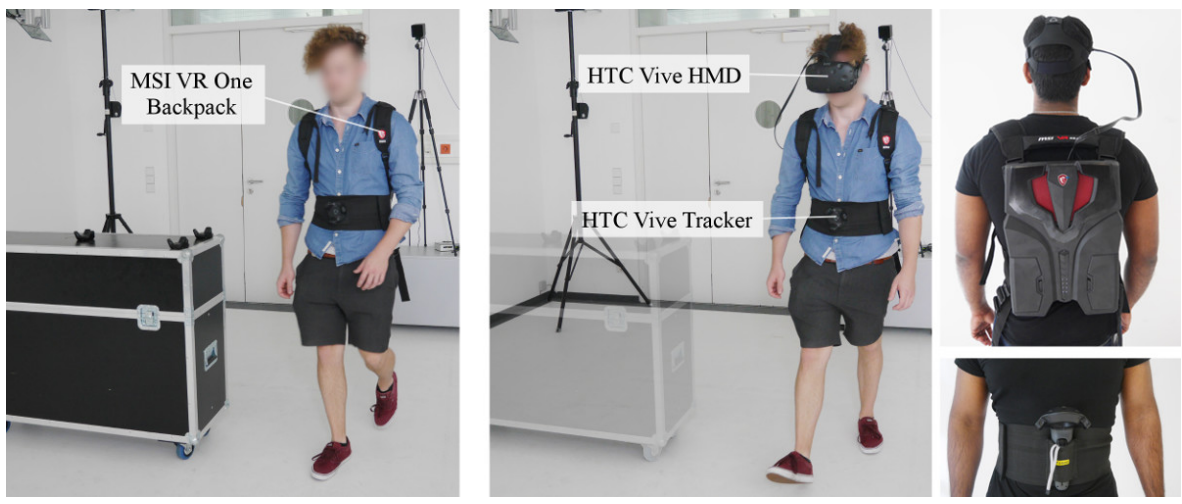

Fig. 1. Representative experimental setup using a HTC Vive controller and an HMD in combination with a VR backpack to compare human locomotion behavior, both in the real world (left) and virtual reality (mid). The right section detailedly depicts the utilized hardware.

assembly processes, which are traditionally validated using physical prototypes, are increasingly assessed in virtual environments using immersive visualization techniques such as head-mounted displays (HMD) [26] or CAVEs [23]. The validity of the gathered results and consequently their significance for production validation, however, is widely unknown - in particular with regard to human locomotion behavior using state-of-the-art HMDs. Consequently, it is crucial to investigate the disparity between walking without any equipment and while being immersed wearing a HMD.

To bridge this gap and to further contribute to a better understanding of human locomotion in virtual environments, this paper presents a study $(n=30)$ analyzing gait in three independent scenarios, replicated both in virtual reality (VR) and in real world (RW). The experiment covers linear walking, non-linear walking, and obstacle avoidance. The results provide novel insights into the effect of immersive simulations on various gait parameters. During the experiment, a HTC Vive connected to a VR backpack PC is used - as depicted in Fig. 1. Special care has been given to design the experiment so that findings are generally valid and can be applied to a wide range of domains beyond production planning.

In particular, the three main contributions of this paper are an analysis of the influence of an HMD-based immersive simulation on ...

(1) ...the velocity, acceleration, and number of steps for linear walking.

(2) ... the turning radius, goodness of fit and shape error for non-linear walking.

(3) ... the clearance distance, goodness of fit and shape error for obstacle avoidance.

The remainder of the paper is structured as follows: First, the state-of-the-art in the context of behavior analysis of human locomotion in virtual worlds is reviewed. Second, three generic experimental scenarios are presented to analyze human gait: Linear walking, non-linear walking and obstacle avoidance. The paper concludes with an assessment and an outlook on further optimizations.

\section{RELATED WORK}

Various research has already been carried out in the field of behavior analysis of human locomotion comparing a virtual with the real world. The following gives an overview of these works. 


\subsection{Locomotion using Dedicated Hardware Devices}

Within the context of immersive navigation in virtual spaces, locomotion interfaces are one of the most commonly used techniques. By using equipment such as joysticks, game pads, treadmills, roller-skates or bicycles [4, 5, 15, 29], a pseudo motion inside the virtual environment is provided to the user - according to the respective steering inputs. Multiple works present various metaphors like the omni-directional treadmill [7], Cyberwalk [32], the Virtusphere [36] and the virtual intuitive striding unit [25].

In the context of locomotion interfaces, various works measuring the correspondence to the real world have been presented. Amongst others, Whitton et al. [38] performed a study analyzing different locomotion interfaces. The authors conclude that the flying metaphor does not correlate with its natural counterpart. Moreover, Nabiyouni et al. [24] point out that locomotion interfaces can improve aspects of user satisfaction (e.g., amusement), however, at the expense of accuracy. These findings are supported by Suma et al. [34]. In essence, it is to be noted that on the one hand such devices decrease the perceived naturalness of walking. On the other hand the gait patterns in VR do not correspond to those exhibited in the real world. Furthermore, the resulting walk paths and velocity profiles significantly depend on the parametrization of the metaphor (e.g., maximum velocity or acceleration). As a consequence, if the goal is to elicit similar human behaviors as in the real world, these methods lack of external validity and thus cannot be applied to the mentioned industrial use-cases.

\subsection{Locomotion using Natural Walking Techniques}

Even though being limited to the size of the tracking area, natural walking techniques are considered to be the most appropriate approach for virtual navigation, since the participants' presence is enhanced by being able to unrestrictedly move through the virtual environment [30,35]. In general, natural walking techniques can be divided into isometric and non-isometric methods [33]. The former utilize a mapping between real and virtual space which preserves distances and angles of a user's movement. For instance, when a person walks one meter, the virtual scene is simultaneously translated one meter. In contrast, non-preserving mappings guide a user on differing paths in RW and VR. A prominent approach is redirected walking [27], whose goal is to manage physical walking space in an intelligent way to allow an exploration of virtual spaces larger than the available physical space. To realize this, the virtual environment is slowly rotated while the person is traveling along a straight path. The user will remain unaware of this redirecting as long as the delta of changes is below a specific perceptual threshold. Moreover, it is possible to travel through VR while remaining in one position in RW. These so-called walking-in-place techniques (e.g., [9]) simulate the sensation of natural walking by means of performing a stepping motion.

In the context of natural walking techniques in VR, only limited research has been carried out investigating their influence on locomotion behavior. Mohler et al. [21] report that participants walked significantly slower with a decreased stride length in VR. Fink et al. [10] and Gerin-Lajoie et al. [11] studied the locomotor behavior when avoiding a stationary obstacle in RW and VR. The authors support the findings made by Mohler et al. and further comment that participants showed an increased clearance distance to obstacles when wearing a HMD. Moreover, their results reveal that the shape of the walk path is not affected by the use of a HMD. More recently, the work presented by Janeh et al. [16] studies natural walking with both isometric and non-isometric mappings for linear walking. The results indicate that isometric mappings tend to have higher validity with less divergence of gait parameters (depending on the velocity of visual self-motion feedback).

\subsection{Assessment of previous work}

While analyzing the relevant aspects of locomotion in virtual and the real world, previous work predominantly focuses on locomotion interfaces and on partial studies, which are not integrated into a coherent framework. The latter includes the analysis of certain aspects of traveling in virtual spaces - such as collision avoidance [10], 
locomotion towards an oriented target [6] or the influence of the field of view (FoV) of the HMD [19, 20]. Furthermore, visualization hardware with a significantly lower display resolution and higher tracking latency compared to the HTC Vive HMD (Edition 2016) is utilized. Consequently, the influence of highly immersive VRsystems on locomotion parameters is currently unknown. Therefore, this work closes this gap by investigating the impact of a HTC Vive system coupled with a wireless VR backpack on virtual walking by unifying and extending previous experimental setups.

\section{OVERVIEW OF EXPERIMENT}

In order to analyze the difference of human locomotion between RW and VR for the use-case of production planning, the experimental setups presented in this paper are derived from a representative workplace within a final assembly line.

\subsection{Deduction of experiment structure}

Fig. 2 sketches the layout of this station stemming from an actual automotive final assembly line. The workplace comprises two racks, one table, a movable trolley and the car being transported from right to left. Moreover, it can be seen that the assembly operator has to successively carry out five assembly tasks (blue dots). Note that tasks number 2 and 5 have to be performed while the car is moving (dotted lines). In order to execute the mentioned assembly tasks, the person has to further walk to the respective location - represented by black arrows. In general, it is to be noted that walking in automotive final assembly lines predominantly takes place in a range up to $3.0 \mathrm{~m}$. Consequently, this range is chosen to be investigated within this paper. Moreover, it can be seen that stations might also contain trolleys, tools or other objects which have to be avoided by the operator (see task 1 and 2). Furthermore, some parts have to be provided in lanes (i.e., points 3 and 4 in Fig. 2), due to their size or logistical concepts.
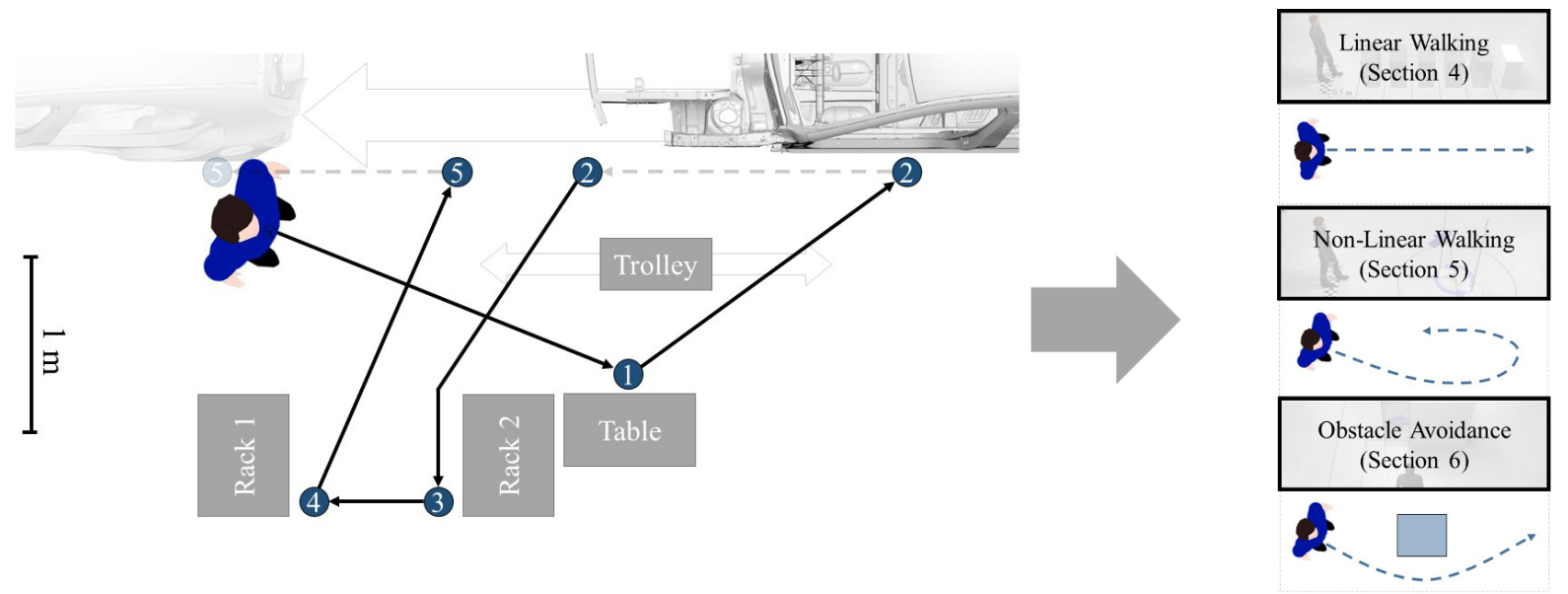

Fig. 2. Left: Representative workplace within a final assembly line and the corresponding assembly tasks; Right: Three cases of path conditions are examined in the experiments.

Based on this analysis, more generally valid experimental setups are derived by means of clustering occurring walk paths into three main types (see right side of Fig. 2): One the one hand side, human locomotion is investigated with regard to linear walking in terms of dynamic gait parameters, such as velocity and acceleration. On the 
Behavior Analysis of Human Locomotion in the Real World and Virtual Reality for the Manufacturing Industry

other hand, the shapes of the resulting trajectories are analyzed in the context of both, non-linear walking and obstacle avoidance.

\subsection{Participants}

In order to draw conclusions about the behavioral differences in RW and VR, a comprehensive group of 30 healthy participants were recruited for the experiment. The group comprised five females and 25 males with an age ranging from 19 to $59(\mu=26.46, \sigma=7.50)$. The heights were between $1.65 \mathrm{~m}$ to $1.90 \mathrm{~m}(\mu=1.78 \mathrm{~m}, \sigma=.07 \mathrm{~m})$, whereas the average body weight was $74.15 \mathrm{~kg}(\sigma=13.09 \mathrm{~kg})$. Furthermore, the population consisted of 23 students and seven production planning employees. Nobody reported vision, balance or perception disorders which could alter the results. Ten individuals wore glasses or contact lenses. 13 persons had previous experience with HMDs and eight were regular gamers. All participants were employees of the car manufacturing company and participated voluntarily. A potential adverse impact on the employment is excluded as the anonymized results are neither accessible to the company nor to third parties (e.g., supervisors or colleagues). Moreover, ethical approval was obtained as per norms from the manufacturing company. Concerning the experiment conducted, ethical principles [31] were considered, and the research strategy was chosen carefully. Furthermore, each individual gave prior consent regarding their participation within the experiment and the use of the anonymous data in further research and publications. Also, all participants were given information regarding the aim, description and the purpose of the experiment and their right to withdraw from the study at any time.

\subsection{Apparatus}

The experiment and all of its scenarios were conducted in a room with the dimensions of $7.0 \mathrm{~m} \times 5.0 \mathrm{~m}$ (ceiling height of $3.0 \mathrm{~m}$ ). To travel through the virtual scene, a HTC Vive HMD (Edition 2016) was utilized due to its high display refresh rate $(90 \mathrm{~Hz})$, large field of view $\left(100^{\circ}\right.$ horizontal, $110^{\circ}$ vertical $)$, and high display resolution (1080 horizontal $\times 1200$ vertical per eye) $[14,20]$. As the aim of this paper is to compare locomotion occurring on the shop floor with VR, participants did not wear equipment restricting the FoV, resolution or update rate in RW. Consequently, a regular binocular field of view of approx. $200^{\circ}$ horizontal and $135^{\circ}$ vertical is assumed for RW [37]. The tracking system covered an area of $4.0 \mathrm{~m} \times 3.0 \mathrm{~m}$ which was centrally aligned in the utilized room. The HMD was further attached to a MSI VR One backpack PC (3.6 kg) equipped with a GTX 1070 graphics card and an Intel Core i7 6820HK with $2.9 \mathrm{GHz}$ (see Fig. 1). Using a backpack, interfering factors related to the wiring of common HMDs could be ruled out.

In order to analyze the locomotion parameters, the position and velocity of each person's center of mass $(\mathrm{CoM})$ was monitored during the experiment. Following Auvinet et al. [1], a controller (200 g) of the HTC Vive tracking system (update rate $500 \mathrm{~Hz}$ on IMU, with drift correction at $60 \mathrm{~Hz}$ ) gathering information with six degrees of freedom was placed on the front side in center of the user's hip at the landmark "umbilicus". The tracker was mounted using an elastic belt fastened around the person's hip as depicted on the right side of Fig. 1. Subsequently, the CoM was individually derived for each participant from this tracking position as the extension of the controller's z-axis while taking into account half of the torso depth and the distance between controller and skin.

Each of the three scenarios comprised varying experimental setups which are detailedly described in sections 4,5 and 6. Regardless of the scenario, a virtual counterpart of the respective experimental setup was modeled using Unity3D. Besides utilizing detailed 3D models of the locomotion target, obstacles and poles, the VR replica also included a reconstructed model of the room, comprising walls, tables, chairs, and windows, as well as closely matched lighting conditions. These efforts were taken to support a comparable optical flow when walking under either immersive condition. Moreover, human locomotion was tracked isometrically, so that the user's movements in physical space were translated 1:1 to VR. 


\subsection{Procedure}

The behavioral disparity in RW and VR was evaluated in three different scenarios (see sections 4, 5 and 6) using repeated measures. Each sub-experiment was successively recorded one week after the previous. In order to rule out systematic effects within each scenario, participants were randomly assigned to two groups. One half started in VR, the other half in RW. Furthermore, the order of the respective locomotion tasks was randomized for each participant. This individual order, however, was kept constant in VR and in RW to ensure similar conditions. In both cases, the participants wore the MSI VR One backpack to counterbalance the effect of carrying the PC on shoulders.

Within each of the scenarios, first, the participant was equipped with the PC, HMD and the tracking devices. Second, the conditions of the experiment were orally explained to each person in a standardized manner. Afterwards, the participant had the possibility to practice the tasks in VR and RW for $5 \mathrm{~min}$ to ensure similar conditions in terms of learning curve and level of practice - following previously introduced procedures [22, 28] Subsequently, locomotion behavior was analyzed in one of the three scenarios, which took between 45 and 75 minutes per person. Note that for each scenario the locomotion task was repeated 10 times, whereas walking was performed at self-selected, natural speed. During the experiment, only the participant and the experimenter (identical person for each scenario) stayed inside the laboratory. Moreover, no communication took place in order to minimize distractions. In general, participants were allowed to take breaks any time or stop the experiment completely if they experienced symptoms of motion sickness. However, no individual reported such problems nor aborted the experiment.

\subsection{Data analysis}

To quantify the influence of the experiment condition on locomotion, the behavior of the overall-group $(n=30)$ in the real world and virtual reality is compared. To achieve this, the 10 trails, being performed by one participant in the context of one condition (e.g., linear walking for $3 \mathrm{~m}$ while wearing the HMD) are averaged. The outcome of this procedure are 30 datasets for VR and RW. Next, a shapiro-wilk test is performed using SPSS, to analyze whether both measurements are normally distributed. If this is the case (for VR and RW), a paired-sample t-test, otherwise, a wilcoxon matched-pairs signed-rank test, is chosen (SPSS). The confidence level for all methods is defined to be $95 \%$. Finally, the test's power is calculated using $G^{*}$ Power [8]. According to G*Power, the group of 30 participant in combination with a required power of 8 allows to statistically significantly prove effect sizes above .476 for the non-parametric and .464 for the parametric test (cutoff-value). Furthermore, non-linear walking and obstacle avoidance (see sections 5 and 6) include a geometrical comparison of the motion corridor and the median trajectory. The former represents the entirety of all trajectories within one experimental condition. To compare the overall-routes, a two-dimensional histogram (bins $=100$ ) is set up for VR and RW. The two resulting histograms are secondly compared in terms of correlation by means of the OpenCV library [3] (version 3.3.0, function compareHist). Using this metric, behavioral differences (e.g., different walking patterns) can be geometrically investigated on a macroscopic scale. In order to further compare routes on a microscopic scale, a median trajectory for each motion corridor is obtained by means of k-nearest neighbor alignment. Next, both routes are compared using dynamic time warping [12]. The resulting normalized error allows to draw conclusions on the walking shape similarity. Table 1 summarizes the outcome of the analysis.

\section{SCENARIO 1: ANALYSIS OF DYNAMIC LOCOMOTION PARAMETERS}

For an initial analysis of the difference of human locomotion between RW and VR, dynamic locomotion parameters are examined. A simple locomotion task (linear walking) is considered in which a participant walks between two points (starting position and obstacle) along a straight line (varying distances) at self-selected walking speed. This scenario comprises two independent variables: The position of the rectangular shaped obstacle (see Fig. 3), 


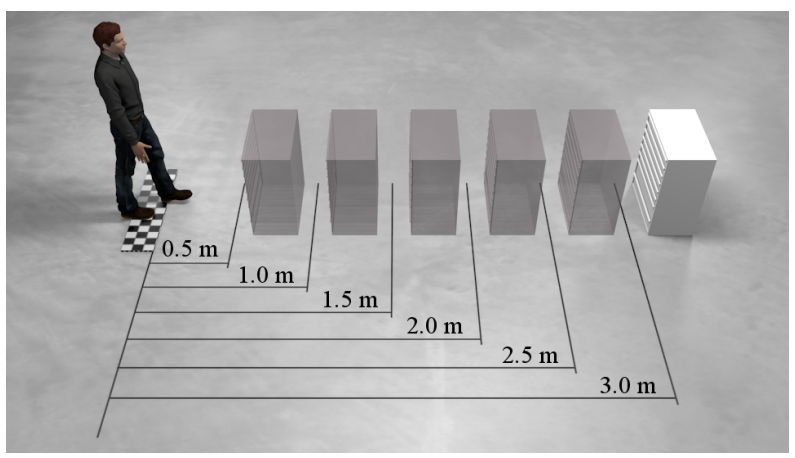

Fig. 3. Experimental setup of experiment 1: Participants walked on a straight line from a starting point to a locomotion target for six distances between $.5 \mathrm{~m}$ and $3.0 \mathrm{~m}$.

which defines the walking distance, and the experiment condition (not immersed / fully immersed). Moreover, the dependent variables for this scenario are chosen to be the magnitudes and profiles of velocity and acceleration, as well as the number of steps.

\subsection{Experimental Setup}

As pictured in Fig. 3, the experimental setup consisted of a ground-leveled starting point and a locomotion target, which was represented by a rolling case cabinet with the dimensions of $1.2 \mathrm{~m} \times .4 \mathrm{~m}$ (height of $.9 \mathrm{~m}$ ). For all varied walk path lengths, this box was positioned $\approx .2 \mathrm{~m}$ behind the actual end point, thus compensating the clearance distance between the person and the object. By using a cabinet instead of a simple end point marked on the floor, realism was increased as the obstacle resembled the trolley in the application domain. In addition to that, this way participants were not forced to tilt their heads downwards to focus on a target near the ground, which could have affected the walking behavior compared to the target scenario. Moreover, the test persons also were not tempted to execute the motion in a restricted way by trying to place their foot exactly on the target mark.

To cover different walking distances, the target box was successively placed at six positions on a straight line of $3.0 \mathrm{~m}$ with a step width of $.5 \mathrm{~m}$. The starting point remained fixed - as depicted in Fig. 3. Consequently, dynamic locomotion parameters, both in RW and VR, could be analyzed in relation to walk path lengths of $.5 \mathrm{~m}, 1.0 \mathrm{~m}$, $1.5 \mathrm{~m}, 2.0 \mathrm{~m}, 2.5 \mathrm{~m}$ and $3.0 \mathrm{~m}$. Each of the six takes included 10 trials of walking between the starting and the target point at the participant's comfortable speed. Having reached the box, the participant returned to the start after standing still for $5 \mathrm{~s}$. This pause was repeated at the starting point before, again, walking to the target. These two pausing phases before and after each locomotion cycle served as distinctive landmarks to reliably detect walking phases.

\subsection{Results}

Using the above described procedure, trajectories with an overall length of $4.52 \mathrm{~km}$ could be yielded, while the overall experiment time for each participant was one hour and fifteen minutes. The following gives an overview on the gathered results.

4.2.1 Velocity. The first row of Fig. 4, illustrates the behavioral differences in terms of maximum velocity for each of the six distances. This value is obtained by means of fitting a second degree polynomial function to the velocity profile. Next, the maximum velocity is defined to be the vertex of the parabola. This intermediate step is necessary, as the utilized least squares method [18] minimizes the influence of velocity-peaks (e.g., due to jerks), 
which otherwise would lead to random errors. Besides analyzing the vertex, the fitted quadratic coefficient is furthermore analyzed, as it reflects the shape of the velocity profile. In particular, high scores indicate a stretched, drawn-out curve, whereas low values point out an acute-angled profile.

Fig. 4 and Table 1 reveal, that the median of maximum velocity is linearly increasing for both experiment conditions from approximately .605 to $1.084 \mathrm{~m} / \mathrm{s}$ and the same applies for the standard deviation. The delta of medians (RW and VR) ranges from -.003 to $-.091 \mathrm{~m} / \mathrm{s}$, while the mean deviates in a similar manner (i.e., -.014 to $-.134 \mathrm{~m} / \mathrm{s}$ ). Moreover, a shapiro-wilk test is performed for each data-set, pointing out normal distributions for all test-cases - except for $3.0 \mathrm{~m}$, while wearing no HMD $\left(p_{\text {shap }}=.048\right)$. Therefore, a paired-sample t-test is utilized for the distances $.5 m-2.5 m$, while three meters are analyzed using a wilcoxon matched-pairs test. The tests reveal no statistical differences for $.5 \mathrm{~m}$, combined with an insufficient test-power of .492. In contrast, the remaining walk path lengths show both, significant statistical differences $\left(p_{\text {si }} g=.001-.005\right)$, adequate $1-\beta$ values - and high effect sizes.

Regarding the shape coefficient, Fig. 4 and Table 1 sketch a similar, however, inverse behavior: It can be seen, that for short walk paths, high quadratic coefficients are reached. This indicates strongly arched velocity-curves for $.5 \mathrm{~m}$. Over the course of walk path lengths, these values (VR and RW) show an inverted exponential decay, which ends at $\approx-.2 \mathrm{~m} / \mathrm{s}^{3}$ (flat parabola). The standard deviation follows a similar curve. The quadratic coefficients are normally distributed for following walk paths: $1.0 \mathrm{~m}-2.5 \mathrm{~m}$ (RW and VR) and $.5 \mathrm{~m} / 3.0 \mathrm{~m}$ for VR. As a consequence, non-parametric tests are chosen for $.5 \mathrm{~m} / 3.0 \mathrm{~m}$, while t-tests are carried out for the remaining cases. The $p$-values indicate differences for each walk path length (except $1.0 \mathrm{~m}$ ), however, a sufficient test-power can only be reached for 2.0 and $3.0 \mathrm{~m}$. The effect sizes are ranging between small and large.

4.2.2 Acceleration. Row three in Fig. 4 depicts the distribution of the mean acceleration in form of boxplots. This score is calculated by means of determining the velocity gradient between the first trajectory element and the maximum velocity (see above). Using this procedure, interferences stemming from both, human gait (e.g., ground reaction forces induced jerks) and the elastic belt, can reliably be eliminated. However, it is to be noted, that this value does not reflect the meta-stable and non-linear acceleration peak during the first gait cycle, but robustly measures the linear growth-factor to the vertex.

In general, the median follows the behavior of the velocity shape, however, in an inverted form. In particular, the score decreases from 1.031 to $.471 \mathrm{~m} / \mathrm{s}^{2}$ over the varied walking tasks. Regarding the deviation between RW and VR it is to be noted, that no clear correlation to the distance can be observed. In contrast, the values range from .094 for $.5 \mathrm{~m}$, to $.041 \mathrm{~m} / \mathrm{s}^{2}$ for $3.0 \mathrm{~m}$. A shapiro-wilk test reveals not normally distributed scores for $.5 \mathrm{~m}$ while wearing no HMD. This leads to the conclusion, that $\mathrm{t}$-tests can be utilized for the remaining distances. Regarding the statistical analysis, significant differences can be observed for $1.5 \mathrm{~m}$ to $3.0 \mathrm{~m}$, while medium and large effect sizes are measured.

4.2.3 Number of Steps. The forth row in Table 1 describes the behavioral differences in terms of number of steps. Regarding the median, it becomes apparent, that for both experiment conditions the number of steps are steadily increasing from $\approx 2.1$ to $\approx 6.1$. Moreover, the delta between VR and RW accounts for approx. a half step. Following a shapiro-wilk test, non-parametric tests are utilized in each case. Even though an evident trend to reduce the number of steps can be observed in RW, the tests' power do not allow a statistical significant conclusion.

\subsection{Discussion}

Regarding the approximation of the velocity using quadratic functions, it is to be noted, that throughout this scenario, parabolic-shaped profiles can be observed. This circumstance can be traced back to the fact, that the rather short distances do not induce walking at constant speed. In contrast, the group of participants is constantly 
Behavior Analysis of Human Locomotion in the Real World and Virtual Reality for the Manufacturing Industry

- $20: 9$
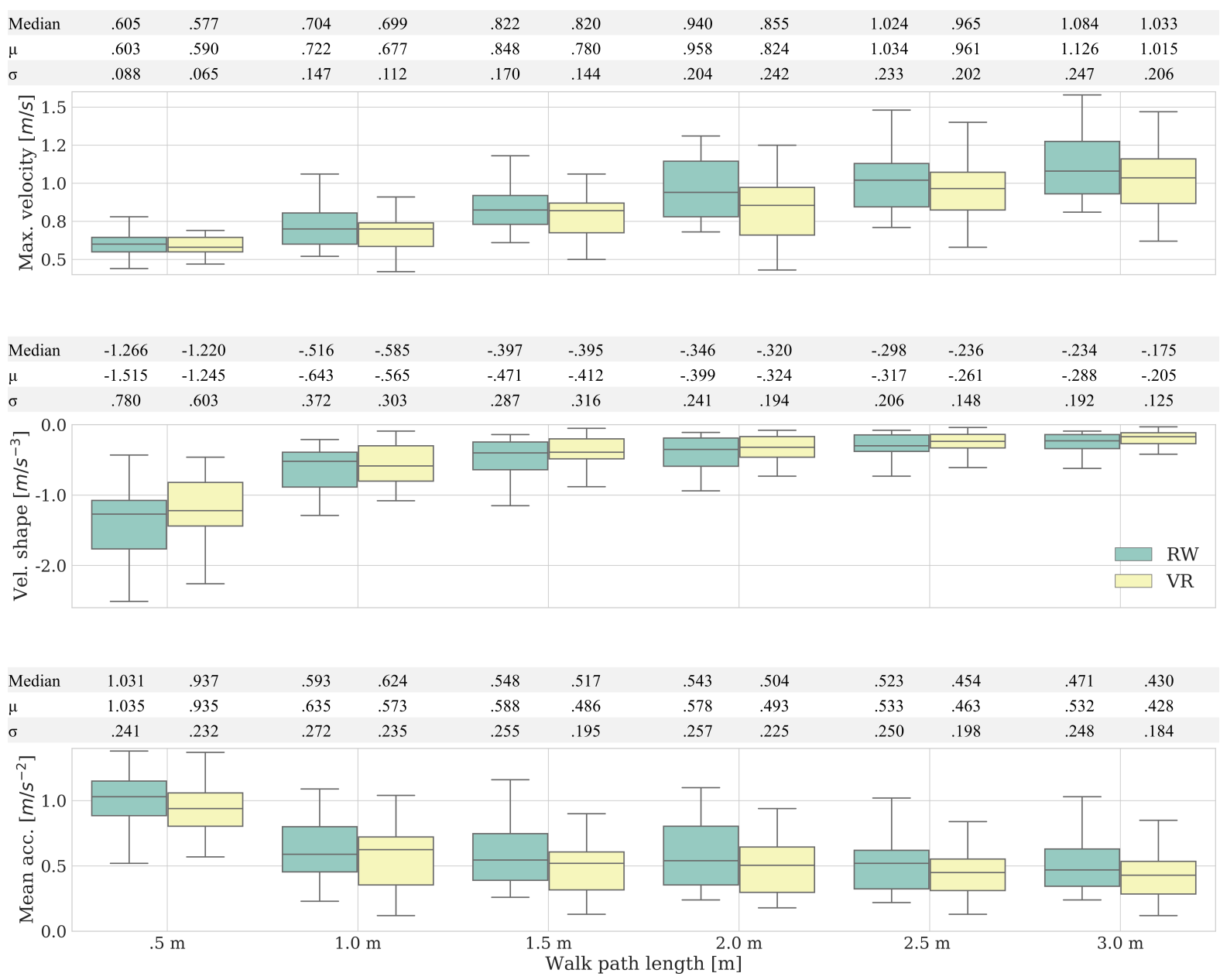

Fig. 4. Overview of maximum velocity (top), velocity shape (mid) and acceleration distribution (bottom) for real world and virtual reality.

de- or accelerating. Fig. 4 supports this assumption, as a steady growth of the maximum velocity can be observed. Constant walking, however, would lead to curves, which flatten out for longer distances. Consequently, quadratic functions seem ideally suited to represent the velocity profiles. To nevertheless measure the applicability of this procedure, the mean squared error (MSE) between each fitted function and the corresponding captured profile is determined. To further compare the outcomes with more elaborate regression models, cubic, quartic and quintic polynomial functions also are fitted to the same dataset. The results reveal, that the quadratic fit scores a MSE of $.017\left(\sigma=.009(\mathrm{~m} / \mathrm{s})^{2}\right)$. For the higher-degree functions, a slight reduction to $.015, .013$ and $.012(\mathrm{~m} / \mathrm{s})^{2}$ can be observed. Same applies for $\sigma$. To further rule out systematic errors for determining the maximum velocity, the mentioned functions' vertexes are calculated: Delta to third-degree $(\mu=.021 / \sigma=.028 \mathrm{~m} / \mathrm{s})$, to fourth-degree $(\mu=.030 / \sigma=.052 \mathrm{~m} / \mathrm{s})$ and to fifth-degree $(\mu=.033 / \sigma=.063 \mathrm{~m} / \mathrm{s})$. Summarizing both findings, it can be 

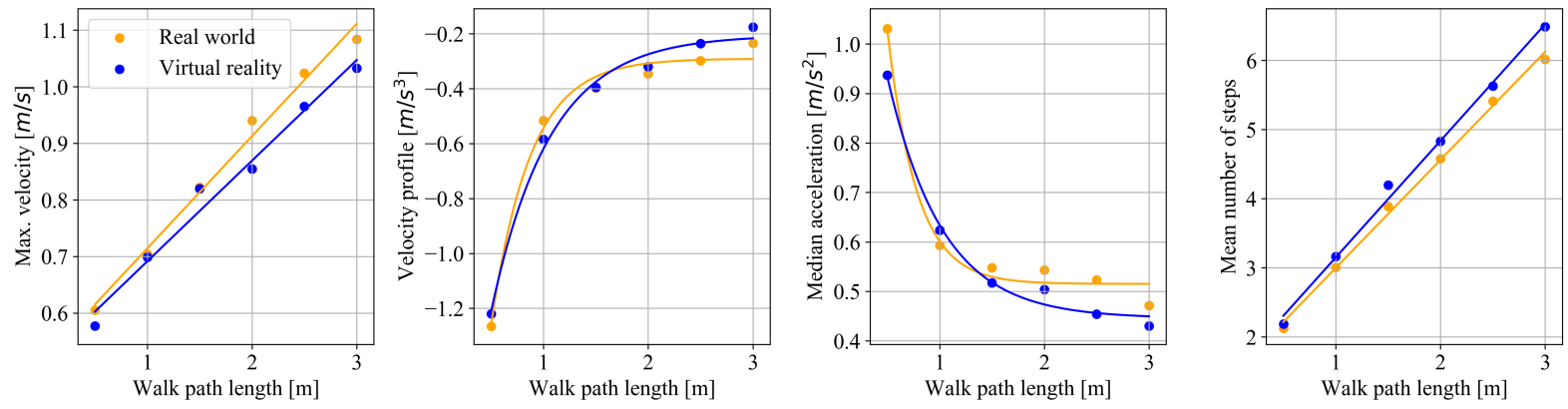

Fig. 5. Regression models of velocity, shape of velocity profile, acceleration and number of steps as a function of walk path distance. Note that the three Y-axes comprise different scales.

concluded that the observed differences can be regarded as small, which verifies the usage of the quadratic function.

Combining velocity, acceleration and number of steps (see Fig. 4 and Table 1) a clear trend towards a reduced performance when wearing a HTC Vive can be observed. In particular, statistically significant differences in terms of maximum velocity for distances $>.5 \mathrm{~m}$ coupled with large effect sizes are found. Overall, participants walked between .04 and $.09 \mathrm{~m} / \mathrm{s}$ slower in VR, which corresponds to a percentage deviation of 5 and $9 \%$. This correlates with the results from Janeh et al. [16], who investigated isometric walking using two experiment conditions. Amongst others, the authors report a significant difference $(p=.004)$ between walking velocity being performed in RW and VR, which could be confined for $>.5 \mathrm{~m}$. This finding is complemented by the mean acceleration, showing a similar pattern. Again, statistically significant deviations can be evidenced for distances $>1.0 \mathrm{~m}$, which are also accompanied by large effect sizes. The median deviation between VR and RW accounts for .031 to $.069 \mathrm{~m} / \mathrm{s}^{2}$, which corresponds to an increased acceleration in RW between 6 and 13\%. In geometrical terms, the acceleration represents the line between the zero crossing and vertex of the velocity parabola. As a consequence, the shape coefficient is indirectly proportional to the derived acceleration. Findings reveal medium to large effect sizes for $>1.0 \mathrm{~m}$, while significant differences for 2 and $3 \mathrm{~m}$ are found. Finally the outcomes coincide with the number of steps executed in RW and in VR: In general, participants tend to take up to $7 \%$ fewer steps in the real world. This corresponds to the velocity delta, since a higher walking velocity is inevitably linked to a higher stride length [2]. Both, the t-test and the effect size support this insight.

Fig. 5 summarizes the differences of locomotion being performed in VR and RW by means of regression models. In particular, the polynomial coefficients are:

- Maximum Velocity $p_{1} \cdot x+p_{0}: p_{1}=.198 \mathrm{~s}^{-1}, p_{0}=.516 \mathrm{~m} / \mathrm{s}$ for RW and $p_{1}=.178 \mathrm{~s}^{-1}, p_{0}=.514 \mathrm{~m} / \mathrm{s}$ for VR.

- Maximum Velocity $p_{2} \cdot e^{-p_{1} \cdot x}+p_{0}: p_{2}=-3.728 \mathrm{~m} / \mathrm{s}, p_{1}=2.690 \mathrm{~m}^{-1}$ and $p_{0}=-.291 \mathrm{~m} / \mathrm{s}$ for RW and $p_{2}=-2.449 \mathrm{~m} / \mathrm{s}, p_{1}=1.776 \mathrm{~m}^{-1}$ and $p_{0}=-.205 \mathrm{~m} / \mathrm{s}$ for VR.

- Acceleration $p_{2} \cdot e^{-p_{1} \cdot x}+p_{0}: p_{2}=3.076 \mathrm{~m} / \mathrm{s}^{2}, p_{1}=3.573$ and $p_{0}=.515 \mathrm{~m} / \mathrm{s}^{2}$ for RW and $p_{2}=1.268 \mathrm{~m} / \mathrm{s}^{2}$, $p_{1}=1.906$ and $p_{0}=.445 \mathrm{~m} / \mathrm{s}^{2}$ for VR.

- Number of steps $p_{1} \cdot x+p_{0}: p_{1}=1.564 m^{-1}, p_{0}=1.433$ for RW and $p_{1}=1.688 m^{-1}, p_{0}=1.462$ for VR.

\section{SCENARIO 2: ANALYSIS OF NON-LINEAR WALKING BEHAVIOR}

Having studied dynamic locomotion parameters in the previous section, the following investigates the influence of the experiment condition on the shape of the resulting CoM trajectories for non-linear walking. For this purpose, 


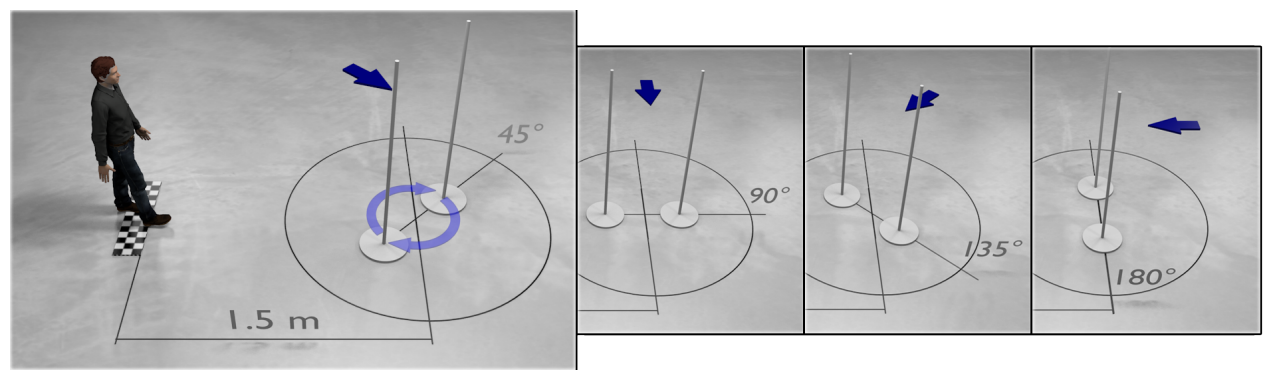

Fig. 6. Experimental setup of scenario 2: Participants walked from a starting position to the target zone between the poles.

a second scenario is conducted in which participants walk through gates with four different orientations relative to the initial walking direction. Similar to the first scenario, this study comprises two independent variables: The orientation of the target (see Fig. 6) and the experimental condition (RW / VR). Moreover, the dependent variables for this experiment are chosen to be spatial shape and the radii of the walk path trajectories.

\subsection{Experimental Setup}

For the evaluation of non-linear walking in virtual environments, a setup inspired by Hicheur et al. [13] was designed, comprising four different spatial configurations. As depicted in Fig. 6, the participants had to walk from the starting point to an oriented target. This target was arranged in four different angular configurations $\left(45^{\circ}, 90^{\circ}, 135^{\circ}, 180^{\circ}\right)$ in order to provoke various trajectory curvatures. As seen in Fig. 6 , the oriented gate consisted of two poles with a height of $2.0 \mathrm{~m}$ and a diameter of $.1 \mathrm{~m}$. The distance from start to the center point of the target was chosen to be $1.5 \mathrm{~m}$. This manipulation can be geometrically interpreted as rotation around the center point. Consequently, the participants performed eight sub-experiments within this section, each containing 10 trials - four for RW and four for VR.

\subsection{Results}

In the second experiment 80 trajectories were recorded for each participant, leading to an experimental time of 45 minutes per person for the whole experiment. Moreover, a total trajectory-length of $7.74 \mathrm{~km}$ was recorded.

5.2.1 Turning radius. Fig. 7 shows the results of the conducted experiment with regard to turning behavior. In particular, the gathered trajectories are examined concerning the radius of curvature, which is estimated by means of fitting a least-squares circle [18]. Table 1 summarizes the descriptive statistics, individually listing all four configurations. Generally, it can be seen that the median values of the radius are ranging from $1.434 \mathrm{~m}$ at $45^{\circ}$ to $.550 \mathrm{~m}$ at $180^{\circ}$ in RW, while in VR the radii are similarly decreasing from $1.369 \mathrm{~m}$ to $.588 \mathrm{~m}$. Moreover, a maximum $\sigma$ can be observed for $45^{\circ}$, which decreases to a nearly constant plateau for the remaining configurations.

For VR, all gathered datasets are normally distributed - according to the shapiro-wilk test. Same applies for $\mathrm{RW}$, except for the $90^{\circ}$ poles-configuration $\left(p_{\text {shap }}=.018\right)$. Consequently, a wilcoxon matched-pairs test is chosen, whereas $45^{\circ}, 135^{\circ}$ and $180^{\circ}$ are compared using a paired-sample t-test. The results reveal no significant differences for $45^{\circ}\left(p_{s i g}=.166\right)$ and $90^{\circ}\left(p_{\text {sig }}=.281\right)$, however, with a low test-power of $.272 / .140$. In contrast, for $135^{\circ}$ $\left(p_{\text {sig }}=.005\right)$ and $180^{\circ}\left(p_{\text {sig }}=.001\right)$ significant differences, coupled with a high power (i.e., .909/.984) can be observed. The effect sizes $d$ correspond to these findings: For the configurations at $45^{\circ}$ and at $90^{\circ}$ the effect size can be considered as small, at $135^{\circ}$ and at $180^{\circ}$ as large.

5.2.2 Trajectory shape. Another important value, which can quantify the differences between VR and RW, is the trajectory shape. Following the methodology described in section 3.5, Fig. 1 displays the two-dimensional 


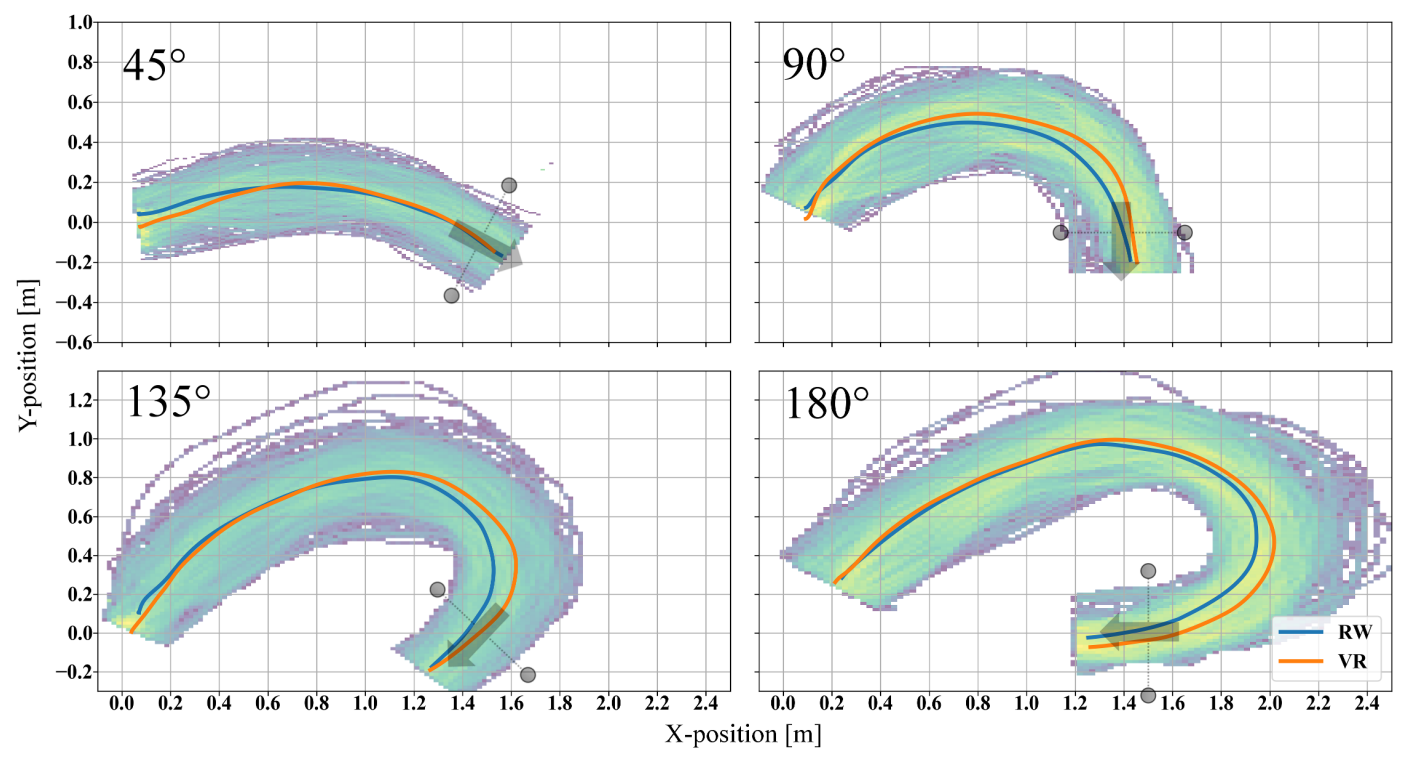

Fig. 7. Two dimensional histograms of the gathered trajectories within the non-linear walking experiment. The green color displays regions which a high frequency, while the blue color correlates to regions with a lower frequency. Moreover, the median trajectories (obtained by nearest neighbor fitting) for VR and RW are visualized.

histograms of all obtained trajectories for the four different scenarios. The correlation values of the motion corridor indicate a high overall-similarity. This supports the visual impression that the motion corridors strongly correspond at a macroscopic scale and cover a similar area. At a microscopic scale (see median trajectory in Fig. 7), however, a different behavior can be identified: For $45^{\circ}$, a low normalized error $e_{n}$ of .012 can be observed, whereas for the remaining pole-configurations, this values rises to $\approx .04$. This circumstance is also reflected in Fig. 7.

\subsection{Discussion}

Analyzing the previously displayed results, it is to be noted that the difference between nonlinear walking in virtual and real world is ambiguous. The high motion corridor correlation leads to the conclusion, that on a macroscopic scale, the 30 participants did not chose a fundamentally varying path. With regard to the turning radius at $45^{\circ}$, no clear conclusion can be drawn, since the gathered scores show a high standard deviation. This is due to the fact, that only a quarter circle with a low curvature serves as an input for the least square fitting. Nevertheless, the low $e_{n}$ deviation points out, that the turning behavior only differs marginally.

In contrast, pole configuration comprising higher angles show an increased delta: For $90^{\circ}$, the quadrupled shape errors in combination with Fig. 7 lead to the conclusion, that participants followed a different path. For both remaining orientations, this trend reinforces. In particular, significant statistical radii difference, sufficient test-power and large effect size can be identified, while the shape errors remain constant. Interestingly, the delta between the median trajectory (VR and RW) jumps from $45^{\circ}$ to $90^{\circ}$, where it remains nearly constant $\left(e_{n}\right.$ values $.045, .042$ and .039). Moreover, in all cases, the median trajectories start to diverge beyond a certain point at which the path curvature rises. As this phenomenon occurs regardless of the respective pole configuration, the 


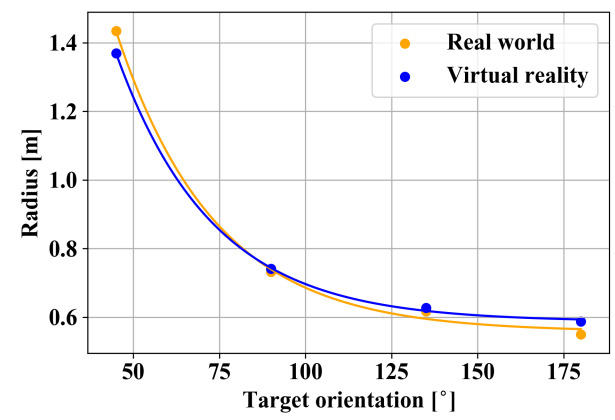

Fig. 8. Exponential function of turning radii as a function of target orientation.

turning angle itself does not seem to strongly influence the positional deviation. Instead a causal connection between angular velocity (corresponds to path curvature) and walk path displacement can be established.

The most likely reason for the high geometrical deviation at $90^{\circ}, 135^{\circ}$ and $180^{\circ}$ is the latency of the HMD. The HTC Vive sensor has a photon-to-motion latency between $11 \mathrm{~ms}$ to $21 \mathrm{~ms}$ (depending on the use of time warping), which is small but still well above a threshold of $5 \mathrm{~ms}$ - as reported by Jerald and Whitton [17] for the minimum noticeable difference of latency during head rotation. Thus, during phases with high head rotational velocity (e.g., experiment $90^{\circ}$ to $180^{\circ}$ ) this delta might induce a delayed turning behavior coupled with a conservative manner of walking. Another possible influence factor is, that the participants might have an increased difficulty to estimate the distances in the virtual environment. Thus, the participants prefer to maneuver themselves so that they approached the poles on a path perpendicular to the line connecting the poles. This is suggested by Fig. 7, as the walk paths in VR have initially a steeper curvature, however, afterwards result in a straight line through the poles. In contrast, in RW a smoother overall turning radius can be observed.

Overall the difference between VR and RW considering the turning radius can be represented by an exponential function as displayed in Fig. 8. The coefficients for $p_{2} \cdot e^{-p_{1} \cdot x}+p_{0}$ are: $p_{2}=4.209 m, p_{1}=-.035$ and $p_{0}=.557 m$ for RW and $p_{2}=3.903 m, p_{1}=-.036$ and $p_{0}=.587 m$ for VR.

\section{SCENARIO 3: ANALYSIS OF CLEARANCE DISTANCE UNDER PRESENCE OF OBSTACLES}

In this last section, the behavioral differences of locomotion in VR and RW are examined under the presence of an obstacle. In particular, the clearance distance between participants and the virtual/real object is in scope of this sub-experiment, whose main idea is inspired by Fink et al.[10]. As described in the previous sections, this study comprises two independent variables: The position of the obstacle (see Fig. 9) and the experiment condition. Moreover, the dependent variables for this experiment are chosen to be trajectory shape, the clearance distance and the goodness of fit between the motion corridors (sum of all trajectories).

\subsection{Experimental Setup}

The experiment comprised a ground-leveled starting point and a box (identical with linear walking), representing the locomotion target. Both locations were set up at a distance of $3.0 \mathrm{~m}$. Additionally, an obstacle was placed half way between the points, which was embodied by a second box with the dimensions of $.4 \mathrm{~m} \times 1.5 \mathrm{~m}$ and a height of $1.8 \mathrm{~m}$ (see Fig. 9), as a proxy of the trolleys used in the assembly line.

In the course of the experiment, the collision avoidance behavior was studied using two different obstacle configurations. In particular, the position of the box was varied orthogonally to the walking direction (see Fig. 9). While in the first configuration the obstacle edge was aligned with the line of sight between start and end (obstacle 


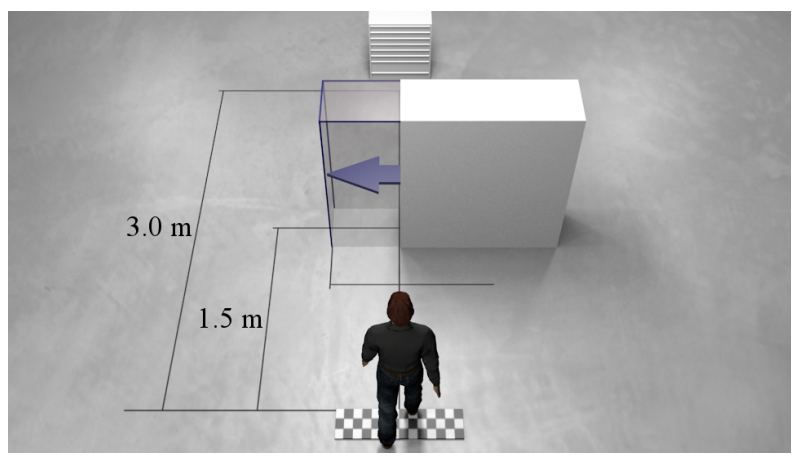

Fig. 9. Experimental setup of experiment 3: Participants walked from a starting point to a locomotion target while avoiding an obstacle being positioned differently in the two conditions.

position 1; overlap $=.0 \mathrm{~m}$ ), in the second configuration the obstacle intersects this line with an overlap of $.5 \mathrm{~m}$ (obstacle position 2). For both scenarios, individuals walked 10 times from start to end with and without HMD, after standing still for $5 \mathrm{~s}$, thus generating a total amount of 40 trials for each individual.

\subsection{Results}

In total, the 30 participants generated a comprehensive database consisting of $5.83 \mathrm{~km}$ walking in both test-cases.

6.2.1 Clearance distance. The clearance distance represents the shortest distance between the participant's center of mass and the obstacle while walking from start to target (SciPy, function cdist [18]). The last row in Table 1 lists the statistics of the gathered results: According to a shapiro-wilk test, the clearance scores are normally distributed, except for the VR / zero overlap configuration. Consequently, the latter is examined using a wilcoxon test, while for the $.5 \mathrm{~m}$ overlap scenario a t-test is chosen. In general, participants kept a median distance of $.419 \mathrm{~m}$ to the box, comprising zero overlap, while walking without a HMD. For an obstacle overlap of $.5 \mathrm{~m}$, this value decreases to $.354 \mathrm{~m}$. While wearing the HTC Vive HMD, values rise to $.437 \mathrm{~m} / .409 \mathrm{~m}$. Comparing RW and VR, participants increased the clearance distances by $4.1 \%$ for zero and $13.4 \%$ for $.5 \mathrm{~m}$ overlap. Table 1 furthermore summarizes the $p$-value for both obstacle position. It can be seen that none of the tests reveals neither significant results $(p=.242 / .132)$ nor reaches sufficient powers (i.e., $1 \beta=.164 / .347)$.

6.2.2 Trajectory shape. Table 1 summarizes the findings of the geometrical comparison of the trajectories. With regard to the correlation between both motion corridors, position 1 scores .777 while the second configuration generates similar outcomes - i.e., .767. It can be ascertained that the shape comparison of the median trajectories (see Fig. 10), reveals a high level of similarity for the zero-overlap configuration, while position 2 results an intermediate delta: $e_{n}$ is $.012 / .024$.

\subsection{Discussion}

Summarizing the presented scenario, the previously indicated overall-influence of the HTC Vive on human locomotion can be further supported. With regard to the clearance distance, only negligible differences between VR and RW can be observed for the zero-overlap configuration. Even though participants slightly increased the median distance by $1.8 \mathrm{~cm}$, the low shape error in combination with the high motion corridor correlation and the statistical tests can exclude a medium or large effect size of the experiment condition on all dependent variables. Fig. 10 further supports these findings due to the high degree of overlap. In contrast, position 2 sketches a slightly different picture: The median trajectory shape error $e_{n}$ doubles, which suggests an influence of this configuration 


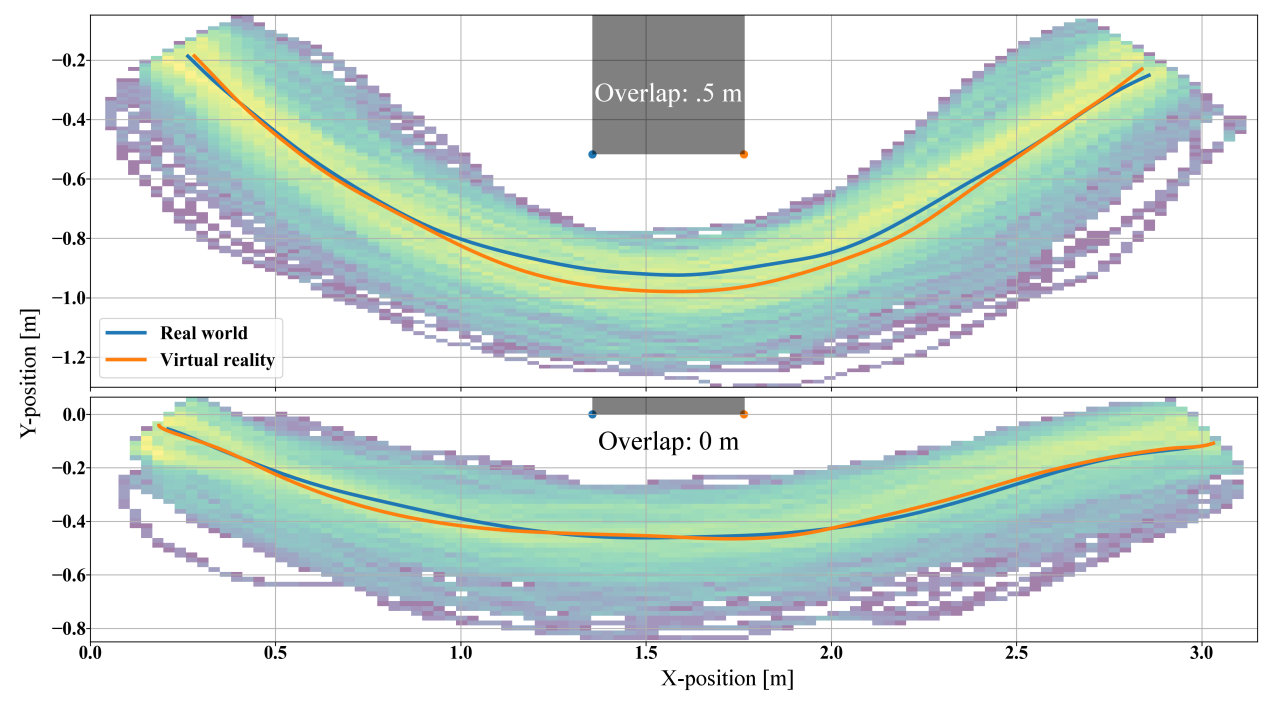

Fig. 10. Two dimensional histograms of experiment 3: The green color displays regions with a high frequency, while the blue color correlates to regions with a lower frequency. In addition, the mean trajectories for VR and RW are visualized.

on the median walk path. However, the effect can only be observed for pos. 2, which mainly differs in terms of turning angle $\left(\approx 60^{\circ}\right)$. As a high angular velocity and the photon-to-motion latency could already be linked to geometrical divergence (see section 5), the displacement of approx. $5.5 \mathrm{~cm}$ can also be mainly attributed to this effect. Interestingly, the normalized shape error of .024 fits well to the line $\left(\approx 60^{\circ}\right)$, connecting the $e_{n}$ values for $45^{\circ}$ and $90^{\circ}$ (i.e., $.012 / .045$ ), which supports this assumption. As consequence, the presence of an obstacle seems to have a small effect on clearance distance in the tested overlap-range, since no clear delta could be found for pos. 1. This finding is underlined by the identical shape error of .012 for both, the $45^{\circ}$ truing scenario and obstacle position 1.

The results partly contradict previous works presented by Fink et al. [10] and Gerin-Lajoie et al. [11] reporting significant higher mean clearance distance deltas of more than $15 \mathrm{~cm}$. Those studies, however, utilized HMDs which comprise a considerably lower FoV of less than $60^{\circ}\left(H T C\right.$ Vive $\left.=110^{\circ}\right)$. Since the vertical field of view is directly linked to the quality of distance judgments (see [19]), the results obtained here can be traced back to the higher technical specifications of the HTC Vive.

The polynomial coefficients $\left(p_{1} \cdot x+p_{0}\right)$ for the clearance distance are: $p_{1, R W}=-.130, p_{0, R W}=.419 \mathrm{~m}$ for RW and $p_{1, V R}=-.056, p_{0, V R}=.437 \mathrm{~m}$ for VR.

\section{DISCUSSION OF ADAPTION AND ORDERING EFFECTS}

In total, the overall duration per person of the presented study accounted for three hours, while half of the time was spend in virtual reality. As the three scenarios were conducted in an identical order, varying levels of VR-experience arose during the course of the experiment. In particular, the differences with regard to time spent in virtual reality accounted for approximately 30 minutes between succeeding sections. To minimize the impact of this effect, the order of the three scenarios was chosen according to their expected sensitivity. Therefore, linear-walking was evaluated in a first step, as dynamic locomotion parameters are most likely to depend on time spent in VR. This assumption is also suggested by previous work (e.g., [22, 28]), reporting adaption effects within the first 3 to 5 minutes. Second and third, turning behavior and obstacle avoidance were analyzed, since it is 


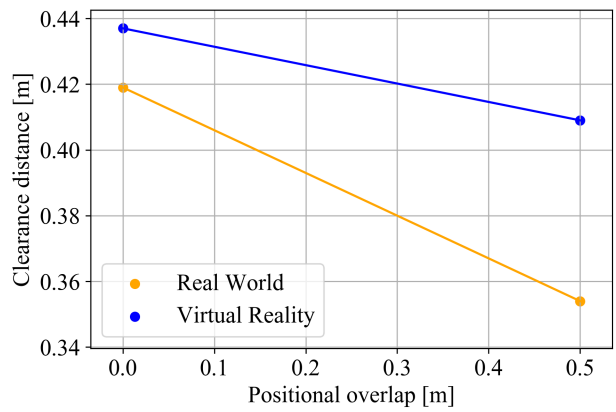

Fig. 11. Linear interpolation of clearance distance as a function of obstacle overlap.

expected that VR-experience only marginally affects the shape of trajectories. Instead, technical specifications of the HMD (e.g., FoV, resolution and photon-to-motion latency) are assumed to have the greatest influence on the geometrical properties.

To analyze the effectiveness of this countermeasure, the correlation between 10 trails, being recorded for one participant in the context of one experimental condition, and their temporal order is determined. Absolute correlation coefficients $>.3$ indicate a not yet settled behavior. For instance, a positive correlation for velocity scores (e.g. $3 \mathrm{~m}$ while wearing the HMD) would point out adaption effects during the 10 trails due to the participant's rising walking speed. In contrast, however, the performed analysis reveals no such findings: Overall, only a negligible correlation can be observed, with coefficients ranging from .163 to -.293 for VR and .218 to -.276 for RW. Both scores are normally distributed according to a shapiro-wilk test ( $p_{\text {shap }}=.760 / p_{\text {shap }}=.317$ ) and show following descriptive statistics: $\mu=-.025 / \sigma=.152$ for virtual reality and $\mu=-.037 / \sigma=0.166$ for real world. As all correlation coefficients are clearly below the threshold of .3, adaption effects within the experimental configurations can be ruled out. Moreover, as the mean correlation for RW and VR tends to zero, adaption effects during the overall-experiment can be excluded with a high probability.

Besides analyzing a potential adaption, the chosen order of scenarios is furthermore examined with regard to takeover-effects. In particular, a potential dependency between $45^{\circ}$ and $90^{\circ}$ turning and $.5 \mathrm{~m}$ obstacle overlap (i.e., $\approx 60^{\circ}$ ) can be identified, since the underlying setups resemble each other. However, as mentioned above, the normalized shape errors of the median trajectory do not show a systematic deviation, but complement each other. Same applies for $45^{\circ}$ and zero overlap. Consequently, no takeover-effects can be observed between nonlinear walking and obstacle avoidance. Furthermore, takeover-effects between linear walking and the remaining scenarios are regarded as unlikely since their setups differ considerably (e.g., $3.0 \mathrm{~m}$ linear walking and $180^{\circ}$ turning).

Summarizing, the analyses show that the experiment is performed in a steady state in combination with no takeover-effects between non-linear walking and obstacle avoidance. A dependency between linear walking and the other scenarios cannot be experimentally excluded, however, it is regarded to be unlikely.

\section{CONCLUSION AND OUTLOOK}

Combining the three scenarios and the findings, derived from an overall-distance of $18.09 \mathrm{~km}$, the experiment reveals an ambivalent picture. In the scenario of linear walking the 30 participants show a clear trend to reduce their walking speed and acceleration in virtual reality (up to $13 \%$ ). Simultaneously, the results reveal an incidental, however, inverted behavior for the number of steps (increase by approx. a half step) which coincides with the reported velocity delta, since a higher walking speed is inevitably linked to a higher stride length. The statistical 
Behavior Analysis of Human Locomotion in the Real World and Virtual Reality for the Manufacturing Industry

- $20: 17$

Table 1. Statistics of the experiment: Mean, median, $p$-value for a Shapiro-Wilk test, and the standard deviation are depicted for RW and VR. Moreover, the effect size, the p-value (t-test or Wilcoxon), the test's power, the motion corridor correlation and the normalized shape error are listed. The color orange highlights $p$-values below .050 .

\begin{tabular}{|c|c|c|c|c|c|c|c|c|c|c|c|c|c|c|c|}
\hline & & & \multicolumn{4}{|c|}{ Real World } & \multicolumn{4}{|c|}{ Virtual Reality } & \multicolumn{3}{|c|}{ Stat. analysis } & \multicolumn{2}{|c|}{$\begin{array}{l}\text { Trajectory } \\
\text { Cor. }\end{array}$} \\
\hline & & Config. & $\mu$ & median & $\sigma$ & $p_{\text {shap }}$ & $\mu$ & median & $\sigma$ & $p_{\text {shap }}$ & $d$ & $p_{\text {sig }}$ & $1-\beta$ & hist. & $e_{n}$ \\
\hline \multirow{24}{*}{ 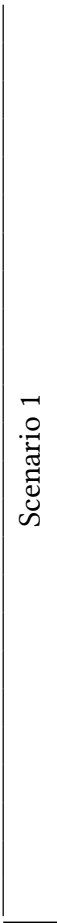 } & \multirow{6}{*}{ 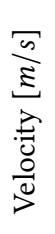 } & $.5 \mathrm{~m}$ & .603 & .605 & .088 & .479 & .590 & .577 & .065 & .209 & .304 & .079 & .492 & - & - \\
\hline & & $1.0 \mathrm{~m}$ & .722 & .704 & .147 & .264 & .677 & 699 & .112 & .344 & .537 & .005 & .890 & - & - \\
\hline & & $1.5 \mathrm{~m}$ & .848 & .822 & .170 & .071 & .780 & .820 & .144 & .602 & .672 & .001 & .974 & - & - \\
\hline & & $2.0 \mathrm{~m}$ & .958 & .940 & .204 & .202 & .824 & .855 & .242 & .196 & .649 & .001 & .966 & - & - \\
\hline & & $2.5 \mathrm{~m}$ & 1.034 & 1.024 & .233 & .080 & .961 & .965 & .202 & .955 & .576 & .003 & .924 & - & - \\
\hline & & $3.0 \mathrm{~m}$ & 1.126 & 1.084 & .247 & .048 & 1.015 & 1.033 & .206 & .998 & .588 & .001 & .923 & - & - \\
\hline & \multirow{6}{*}{ 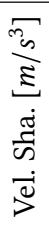 } & $.5 \mathrm{~m}$ & -1.515 & -1.266 & .780 & .000 & -1.245 & -1.220 & .603 & .003 & .339 & .033 & .552 & - & - \\
\hline & & $1.0 \mathrm{~m}$ & -.643 & -.516 & .372 & .114 & -.565 & -.585 & .303 & .169 & .223 & .143 & .326 & - & - \\
\hline & & $1.5 \mathrm{~m}$ & -.471 & -.397 & .287 & .123 & -.412 & -.395 & .316 & .382 & .423 & .025 & .732 & - & - \\
\hline & & $2.0 \mathrm{~m}$ & -.399 & -.346 & .241 & .084 & -.324 & -.320 & .194 & .322 & .570 & .003 & .920 & - & - \\
\hline & & $2.5 \mathrm{~m}$ & -.317 & -.298 & .206 & .056 & -.261 & -.236 & .148 & .357 & .460 & .012 & .793 & - & - \\
\hline & & $3.0 \mathrm{~m}$ & -.288 & -.234 & .192 & .013 & -.205 & -.175 & .125 & .527 & .475 & .004 & .805 & - & - \\
\hline & \multirow{6}{*}{ 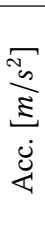 } & $.5 \mathrm{~m}$ & 1.035 & 1.031 & .241 & .045 & .935 & .937 & .232 & .972 & .361 & .024 & .595 & - & - \\
\hline & & $1.0 \mathrm{~m}$ & .635 & .593 & .272 & .481 & .573 & .624 & .235 & .473 & .325 & .110 & .537 & - & - \\
\hline & & $1.5 \mathrm{~m}$ & .588 & .548 & .255 & .069 & .486 & .517 & .195 & .548 & .623 & .006 & .954 & - & - \\
\hline & & $2.0 \mathrm{~m}$ & .578 & .543 & .257 & .179 & .493 & .504 & .225 & .531 & .677 & .002 & .976 & - & - \\
\hline & & $2.5 \mathrm{~m}$ & .533 & .523 & .250 & .093 & .463 & .454 & .198 & .802 & .470 & .034 & .807 & - & - \\
\hline & & $3.0 \mathrm{~m}$ & .532 & .471 & .248 & .134 & .428 & .430 & .184 & .982 & .644 & .004 & .964 & - & - \\
\hline & \multirow{6}{*}{ 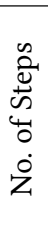 } & $.5 \mathrm{~m}$ & 2.123 & 2.333 & .314 & .000 & 2.187 & 2.000 & .540 & .000 & .149 & .281 & .193 & - & - \\
\hline & & $1.0 \mathrm{~m}$ & 3.006 & 3.000 & .697 & .000 & 3.162 & 3.000 & .753 & .001 & .123 & .259 & .158 & - & - \\
\hline & & $1.5 \mathrm{~m}$ & 3.889 & 4.000 & .849 & .002 & 4.198 & 4.000 & .927 & .001 & .450 & .006 & .759 & - & - \\
\hline & & $2.0 \mathrm{~m}$ & 4.578 & 4.667 & .961 & .001 & 4.828 & 5.000 & 1.064 & .009 & .347 & .028 & .566 & - & - \\
\hline & & $2.5 \mathrm{~m}$ & 5.411 & 5.167 & 1.093 & .001 & 5.629 & 5.667 & 1.193 & .033 & .342 & .031 & .556 & - & - \\
\hline & & $3.0 \mathrm{~m}$ & 6.017 & 6.000 & 1.253 & .032 & 6.488 & 6.333 & 1.395 & .253 & .404 & .013 & .679 & - & - \\
\hline \multirow{4}{*}{ 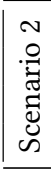 } & \multirow{4}{*}{ 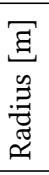 } & $45^{\circ}$ & 1.374 & 1.434 & .310 & .173 & 1.304 & 1.369 & .259 & .056 & .194 & .166 & .272 & .721 & .012 \\
\hline & & $90^{\circ}$ & .726 & .733 & .064 & .018 & .731 & .741 & .056 & .548 & .106 & .281 & .140 & .788 & .045 \\
\hline & & $135^{\circ}$ & .614 & .617 & .043 & .165 & .635 & .627 & .051 & .961 & .557 & .005 & .909 & .708 & .042 \\
\hline & & $180^{\circ}$ & .553 & .550 & .035 & .870 & .591 & .588 & .060 & .869 & .710 & .001 & .984 & .608 & .039 \\
\hline \multirow{2}{*}{ 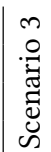 } & $\Xi$ & $0 \mathrm{~m}$ & .434 & .419 & .086 & .519 & .447 & .437 & .087 & .015 & .128 & .242 & .164 & .777 & .012 \\
\hline & $\frac{\bar{\Xi}}{U}$ & $.5 \mathrm{~m}$ & .378 & .354 & .079 & .199 & .401 & .409 & .067 & .588 & .234 & .132 & .347 & .767 & .024 \\
\hline
\end{tabular}

analysis underlines this findings, pointing out significant differences for distances $>1 \mathrm{~m}$ with an adequate test-power. In contrast, no significant differences for non-linear walking can be found for a tuning of $45^{\circ}$. The situation changes for rising angles. In particular, statistically significant differences can be observed for $>90^{\circ}$, reporting large effect sizes for the fitted turning radii. Moreover, an evident delta in terms of trajectory shape can be unveiled. Finally, obstacle avoidance does not reveal significant differences with regard to the clearance distance. In particular, it is expected that the latency of the HMD has a higher impact on the clearance distance as on the perception of distances. 
To transfer these findings to the initially mentioned workplace and the corresponding assembly tasks (see Fig. 2), each of the five walk paths have to be mapped individually to the three use-case independent experiments. The route from start to assembly point 1 has to be regarded as unrestricted linear walking of a distance of $2.35 \mathrm{~m}$. Consequently, the delta for this task can be approximated using the velocity regression model of experiment 1 (see Fig. 5). Same applies for route 1 to $2(1.95 \mathrm{~m})$, since no significant differences in terms of obstacle avoidance could be observed. The counterpart between point 2 and $3(2.14 \mathrm{~m})$ refers to Fig. 5 , since experiment 2 reveals no considerable delta for orientations smaller than $90^{\circ}$. The last two tasks $(.72 \mathrm{~m} / 2.06 \mathrm{~m})$ have to be regard as unrestricted linear walking. Summarizing all five routes, the overall estimated median walking time in the real world accounts for $20.307 \mathrm{~s}$, whereas the identical configuration yields $21.266 \mathrm{~s}$ while wearing an HMD. Assuming an exemplary full cycle time of $100 \mathrm{~s}$ including all assembly tasks, the delta currently comprises only $-4.726 \%$. Contrasted with the considerable benefits of Virtual Manufacturing, namely, the possibility to easily assesses an exhaustive number of variants while using no costly physical hardware, this is considered as practically acceptable. Moreover, based on the regression analysis, this delta can be compensated by extrapolation - as depicted in Fig. 5.

Summarizing all aforementioned findings, it can b e concluded that a medium delta between locomotion being performed in a virtual space and in RW exists given the external conditions realized in the experiment (improved hardware, replicated 3D environment). These new findings partly contradict previous work, reporting a considerable deviation (for instance clearance distance [10]). This can on the one hand be attributed to the use of a HTC Vive due to the significant better technical specifications (e.g., higher field of view, lower latency). On the other hand, another significant differentiator is the use of a backpack PC since it cannot be ruled out that previous solutions (e.g., a second person carrying the wiring set [16]) could have affected the participant's behavior. Consequently, drawbacks being related to the wiring of the HMD, which showed to be a major hurdle for unrestricted walking, could be compensated for the first time. Moreover, since the measured delta decreased in the temporal context of previous work, it is expected that with the ongoing development and simultaneously the increasing level of immersion, this deviation probably will converge towards zero within the next years (e.g., wireless transmitters).

\section{REFERENCES}

[1] Bernard Auvinet, Gilles Berrut, Claude Touzard, Laurent Moutel, Nadine Collet, Denis Chaleil, and Eric Barrey. 2002. Reference data for normal subjects obtained with an accelerometric device. Gait \& Posture 16, 2 (Oct. 2002), 124-134.

[2] Joshua Bailey, Tiffany Mata, and John Mercer. 2017. Is the relationship between stride length, frequency, and velocity influenced by running on a treadmill or overground? International journal of exercise science 10, 7 (2017), 1067.

[3] G. Bradski. 2000. The OpenCV Library. Dr. Dobb's fournal of Software Tools (2000).

[4] Frederick P. Brooks, Jr. 1987. Walkthrough - a Dynamic Graphics System for Simulating Virtual Buildings. In Proceedings of the 1986 Workshop on Interactive 3D Graphics (I3D '86). ACM, New York, NY, USA, 9-21. https://doi.org/10.1145/319120.319122

[5] R. R. Christensen, J. M. Hollerbach, Y. Xu, and S. G. Meek. 2000. Inertial-Force Feedback for the Treadport Locomotion Interface. Presence 9, 1 (Feb 2000), 1-14. https://doi.org/10.1162/105474600566574

[6] Gabriel Cirio, Anne-Helene Olivier, Maud Marchal, and Julien Pettre. 2013. Kinematic Evaluation of Virtual Walking Trajectories. IEEE Transactions on Visualization and Computer Graphics 19, 4 (April 2013), 671-680. https://doi.org/10.1109/TVCG.2013.34

[7] Rudolph P. Darken, William R. Cockayne, and David Carmein. 1997. The Omni-directional Treadmill: A Locomotion Device for Virtual Worlds. In Proceedings of the 10th Annual ACM Symposium on User Interface Software and Technology (UIST '97). ACM, New York, NY, USA, 213-221. https://doi.org/10.1145/263407.263550

[8] Franz Faul, Edgar Erdfelder, Albert-Georg Lang, and Axel Buchner. 2007. G* Power 3: A flexible statistical power analysis program for the social, behavioral, and biomedical sciences. Behavior research methods 39, 2 (2007), 175-191.

[9] J. Feasel, M. C. Whitton, and J. D. Wendt. 2008. LLCM-WIP: Low-Latency, Continuous-Motion Walking-in-Place. In 2008 IEEE Symposium on 3D User Interfaces. 97-104. https://doi.org/10.1109/3DUI.2008.4476598

[10] Philip W. Fink, Patrick S. Foo, and William H. Warren. 2007. Obstacle avoidance during walking in real and virtual environments. ACM Transactions on Applied Perception 4, 1 (Jan. 2007), 2-es. https://doi.org/10.1145/1227134.1227136 
Behavior Analysis of Human Locomotion in the Real World and Virtual Reality for the Manufacturing Industry

[11] Martin Gerin-Lajoie, Carol L. Richards, Joyce Fung, and Bradford J. McFadyen. 2008. Characteristics of personal space during obstacle circumvention in physical and virtual environments. Gait \& Posture 27, 2 (2008), 239 - 247. https://doi.org/10.1016/j.gaitpost.2007.03.015

[12] Toni Giorgino. 2009. Computing and Visualizing Dynamic Time Warping Alignments in R: The dtw Package. fournal of Statistical Software, Articles 31, 7 (2009), 1-24. https://doi.org/10.18637/jss.v031.i07

[13] Halim Hicheur, Quang-Cuong Pham, Gustavo Arechavaleta, Jean-Paul Laumond, and Alain Berthoz. 2007. The formation of trajectories during goal-oriented locomotion in humans. I. A stereotyped behaviour: Trajectories in locomotion I: stereotypy. European fournal of Neuroscience 26, 8 (Oct. 2007), 2376-2390. https://doi.org/10.1111/j.1460-9568.2007.05836.x

[14] HTC. 2017. Discover Virtual Reality Beyond Imagination. (2017). http://www.vive.com/

[15] Kazuaki Iwata, Masahiko Onosato, Koji Teramoto, and Suguru Osaki. 1997. Virtual Manufacturing Systems as Advanced Information Infrastructure for Integrating Manufacturing Resources and Activities. CIRP Annals - Manufacturing Technology 46, 1 (1997), 335 - 338 https://doi.org/10.1016/S0007-8506(07)60837-3

[16] Omar Janeh, Eike Langbehn, Frank Steinicke, Gerd Bruder, Alessandro Gulberti, and Monika Poetter-Nerger. 2017. Walking in Virtual Reality: Effects of Manipulated Visual Self-Motion on Walking Biomechanics. In ACM Transactions on Applied Perception (TAP). 12:1-12:15. http://basilic.informatik.uni-hamburg.de/Publications/2017/JLSBGP17

[17] J. Jerald and M. Whitton. 2009. Relating Scene-Motion Thresholds to Latency Thresholds for Head-Mounted Displays. In 2009 IEEE Virtual Reality Conference. 211-218. https://doi.org/10.1109/VR.2009.4811025

[18] Eric Jones, Travis Oliphant, Pearu Peterson, et al. 2017. SciPy: Open source scientific tools for Python. (2017). http://www.scipy.org/

[19] J. Adam Jones, David M. Krum, and Mark T. Bolas. 2016. Vertical Field-of-View Extension and Walking Characteristics in Head-Worn Virtual Environments. ACM Trans. Appl. Percept. 14, 2, Article 9 (Oct. 2016), 17 pages. https://doi.org/10.1145/2983631

[20] Jonathan W. Kelly, Lucia A. Cherep, and Zachary D. Siegel. 2017. Perceived Space in the HTC Vive. ACM Trans. Appl. Percept. 15, 1, Article 2 (July 2017), 16 pages. https://doi.org/10.1145/3106155

[21] Betty J Mohler, Jennifer L Campos, M Weyel, and Heinrich H Bülthoff. 2007. Gait parameters while walking in a head-mounted display virtual environment and the real world. In Proceedings of Eurographics. 85-88.

[22] Betty J. Mohler, Sarah H. Creem-Regehr, and William B. Thompson. 2006. The influence of feedback on egocentric distance judgments in real and virtual environments. ACM Press, 9. https://doi.org/10.1145/1140491.1140493

[23] T.S. Mujber, T. Szecsi, and M.S.J. Hashmi. 2004. Virtual reality applications in manufacturing process simulation. fournal of Materials Processing Technology 155-156 (2004), 1834 - 1838. https://doi.org/10.1016/j.jmatprotec.2004.04.401 Proceedings of the International Conference on Advances in Materials and Processing Technologies: Part 2.

[24] Mahdi Nabiyouni and Doug A. Bowman. 2015. An Evaluation of the Effects of Hyper-Natural Components of Interaction Fidelity on Locomotion Performance in Virtual Reality. In ICAT-EGVE 2015 - International Conference on Artificial Reality and Telexistence and Eurographics Symposium on Virtual Environments, Masataka Imura, Pablo Figueroa, and Betty Mohler (Eds.). The Eurographics Association. https://doi.org/10.2312/egve.20151325

[25] Toshikazu Ohshima, Ryuki Shibata, Hotaru Edamoto, and Nozomi Tatewaki. 2016. Virtual ISU: Locomotion Interface for Immersive VR Experience in Seated Position (1). In SIGGRAPH ASIA 2016 Posters (SA '16). ACM, New York, NY, USA, Article 18, 2 pages.

[26] Michael Otto, Michael Prieur, Philipp Agethen, and Enrico Rukzio. 2016. Dual Reality for Production Verification Workshops: A Comprehensive Set of Virtual Methods. Procedia CIRP 44 (2016), 38-43. https://doi.org/10.1016/j.procir.2016.02.140

[27] Sharif Razzaque. 2005. Redirected Walking. Ph.D. Dissertation. Chapel Hill, NC, USA. Advisor(s) Brooks,Jr., Fredrick P. AAI3190299.

[28] Roy A. Ruddle, Ekaterina Volkova, and Heinrich H. Buelthoff. 2013. Learning to walk in virtual reality. ACM Transactions on Applied Perception 10, 2 (May 2013), 1-17. https://doi.org/10.1145/2465780.2465785

[29] Mel Slater, Anthony Steed, and Martin Usoh. 1995. The Virtual Treadmill: A Naturalistic Metaphor for Navigation in Immersive Virtual Environments. Springer Vienna, Vienna, 135-148. https://doi.org/10.1007/978-3-7091-9433-1_12

[30] Mel Slater, Martin Usoh, and Anthony Steed. 1995. Taking Steps: The Influence of a Walking Technique on Presence in Virtual Reality. ACM Trans. Comput.-Hum. Interact. 2, 3 (Sept. 1995), 201-219. https://doi.org/10.1145/210079.210084

[31] Deborah Smith. 2003. Five principles for research ethics. Monitor on psychology 34, 1 (2003), 56.

[32] Jan L. Souman, P. Robuffo Giordano, Martin Schwaiger, Ilja Frissen, Thomas Thuemmel, Heinz Ulbrich, A. De Luca, Heinrich H. Buelthoff, and Marc O. Ernst. 2011. CyberWalk: Enabling unconstrained omnidirectional walking through virtual environments. ACM Transactions on Applied Perception (TAP) 8, 4 (2011), 25. http://dl.acm.org/citation.cfm?id=2043607 00061.

[33] Frank Steinicke, Yon Visell, and Jennifer Campos. 2013. Human Walking in Virtual Environments: Perception, Technology, and Applications. Springer New York, New York NY. http://dx.doi.org/10.1007/978-1-4419-8432-6

[34] E. Suma, S. Finkelstein, M. Reid, S. Babu, A. Ulinski, and L. F. Hodges. 2010. Evaluation of the Cognitive Effects of Travel Technique in Complex Real and Virtual Environments. IEEE Transactions on Visualization and Computer Graphics 16, 4 (July 2010), 690-702.

[35] Martin Usoh, Kevin Arthur, Mary C. Whitton, Rui Bastos, Anthony Steed, Mel Slater, and Frederick P. Brooks, Jr. 1999. Walking Walkingin-place Flying, in Virtual Environments. In Proceedings of the 26th Annual Conference on Computer Graphics and Interactive Techniques (SIGGRAPH '99). ACM Press/Addison-Wesley Publishing Co., New York, NY, USA, 359-364. https://doi.org/10.1145/311535.311589

[36] Inc Virtusphere. 2013. Virtusphere. (2013). http://www.virtusphere.com/ 
[37] Bruce A. Whitehead. 1981. James J. Gibson: The ecological approach to visual perception. Boston: Houghton Mifflin, 1979, 332 pp. Behavioral Science 26, 3 (1981), 308-309. https://doi.org/10.1002/bs.3830260313

[38] Mary Whitton, Joseph Cohn, J Feasel, P Zimmons, S Razzaque, S.J. Poulton, B McLeod, and Frederick Brooks, Jr. 2005. Comparing VE locomotion interfaces. (04 2005), 123-130.

ACM Transactions on Applied Perception, Vol. 15, No. 3, Article 20. Publication date: July 2018. 